\title{
Numerical Quadrature and Asymptotic Expansions
}

\author{
By J. N. Lyness $†$ and B. W. Ninham
}

1. Introduction. If a function has a singularity of any type on or near the interval of integration, the conventional methods of numerical quadrature based on polynomial interpolation are difficult to use effectively. Special methods exist for functions having particular singularities. The object of this and a later paper is to develop a simple unified method capable of dealing with a wide class of functions. This class includes all complex analytic functions which do not have essential singularities as well as those whose essential singularities are not too close to the integration interval. The technique which we advocate takes explicit account of algebraic and logarithmic singularities to obtain a complete asymptotic expansion of the error functional. In the case of trapezoidal rules this is a power series in $1 / \mathrm{m}^{2}$ where $m$ is the number of function evaluations. We show that for integrands of the class mentioned above the form of the expansion can be written down by inspection. Is in the Euler-Maclaurin summation formula which is a special case of such an expansion, the coefficients depend on the derivatives at the endpoints and are in general generalised zeta functions or linear combinations thereof.

To indicate further the principal features of the method, we consider the integral

$$
I f=\int_{0}^{1} f(t) d t=\int_{0}^{1} t^{-3 / 4}(1-t)^{-1 / 2} h(t) d t
$$

where $h(t)$ and all its derivatives are continuous in $0 \leqq t \leqq 1$. We use the approximation obtained from the midpoint trapezoidal rule, defined by

$$
R^{[m, 0]} f=\frac{1}{m} \sum_{j=1}^{m} f\left(\frac{2 j-1}{2 m}\right) .
$$

If $f(x)$ were a function without singularities in the closed interval $[0,1]$ the error involved could be written down using the modified Euler-Maclaurin expansion

$$
\begin{aligned}
E f=R^{[m, 0]} f-I f= & -\frac{1}{24 m^{2}}\left[f^{(1)}(1)-f^{(1)}(0)\right] \\
& +\frac{7}{5760 m^{4}}\left[f^{(3)}(1)-f^{(3)}(0)\right]+\cdots .
\end{aligned}
$$

We show in Sections 4 and $j$ of this paper that integration rules which employ polynomial approximation are based on the assumption that the error functional $E f$ always admits an asymptotic expansion of precisely this form.

In the example Eq. (1.1) above, the expansion (1.3) is of course not valid, and such rules are not particularly efficient. In Section 7 we determine the appropriate

Received January 24, 1966. Revised June 20, 1966.

$\dagger$ Present address: Mathematics Division, O.R.N.L., Oak Ridge, Tennessee. 
expansion $E f$, which in this case takes the form

$$
\begin{aligned}
R^{[m, 0]} f-I f & =\frac{a_{0}}{m^{1 / 4}}+\frac{a_{1}}{m^{5 / 4}}+\frac{a_{2}}{m^{9 / 4}}+O\left(m^{-13 / 4}\right) \\
& +\frac{b_{0}}{m^{1 / 2}}+\frac{b_{1}}{m^{3 / 2}}+\frac{b_{2}}{m^{5 / 2}}+O\left(m^{-7 / 2}\right) .
\end{aligned}
$$

In applications expansions such as this may be used as a basis for Romberg integration. Alternatively, since expressions for $a_{s}$ and $b_{s}$ are available, the expansion may be used directly. A judicious combination of both of these approaches is also possible.

The essence of this method of quadrature is to treat Eq. (1.4) as an equation, all of whose terms can be computed except the one unknown $I f$. In the conventional approach, one regards $R^{\left[m,{ }^{01}\right.} f$ as an estimate for $I f$ with a (small) error $E f$. By our approach, it is of no consequence whether $R^{[m, 0]} f$ is a good estimate or not for $I f$. Eq. (1.6) fails to be an identity in the general sense of the term only in as much as the right-hand side is the sum of two asymptotic expansions. For a sufficiently large $m$, which in practice is surprisingly small, each of these expansions has the characteristic property that successive terms at first decrease in magnitude, and then increase. In certain cases there is some justification for truncating this expansion (usually after its smallest term) and for then assuming that the error is smaller than the final included term and of opposite sign. However, this is not generally true even in simple nonpathological cases. Thus the final term can only be used as a rough guide in this context. Truncation of this type is usually justified only in the sense of polynomial approximation.

The work is arranged as follows: In Section 2 we introduce a simple generalisation of Poisson's summation formula which is fundamental to the subsequent work. In Sections 3-5 we treat conventional quadrature methods for functions without important singularities from this point of view. In the remainder of this paper we investigate and classify various asymptotic expansions which arise when singularities occur on the interval of integration. Those with which we are principally concerned here are generalisations of the traditional Euler-Maclaurin expansion. In a forthcoming paper we shall deal with functions with singularities close to the interval of integration.

2. The Fundamental Summation Formula. One form of the Fourier theorem (of Whittaker and Watson [2]) may be stated as follows: If $f(x)$ exists in the interval $[0,1]$ except at a finite number of distinct values of $x$ and

(i) $f(x)=f(x+1)$ for all values of $x$ for which $f(x)$ exists,

(ii) $\lim _{\epsilon \rightarrow 0} \frac{1}{2}[f(x+\epsilon)+f(x-\epsilon)]=f(x)$ for all values of $x$ for which $f(x)$ exists,

(iii) $\int_{0}^{1} f(t) d t$ exists and if the integral is improper, is absolutely convergent, then

$$
f(x)=\sum_{r=-\infty}^{\infty} \exp (-2 \pi i r x) \int_{0}^{1} f(t) \exp (2 \pi i r t) d t
$$

for all values of $x$ for which $f(x)$ exists. We note that there is no condition of bounded 
variation applied to $f(x)$. It follows by trivial rearrangement that

$$
f\left(t_{j}\right)-\int_{0}^{1} f(t) d t=\sum_{r=-\infty}^{\infty} \exp \left(-2 \pi i r t_{j}\right) \int_{0}^{1} f(t) \exp (2 \pi i r t) d t
$$

where the prime on the summation sign denotes omission of the term in the sum for which $r=0$. If further we add linear combinations of this with different values of $t_{j}$ and corresponding weights $a_{j}, j=1,2, \cdots, m$, we have

$$
\sum_{j=1}^{m} a_{j} f\left(t_{j}\right)-\int_{0}^{1} f(t) d t=\sum_{r=-\infty}^{\infty}\left\{\sum_{j=1}^{m} a_{j} \exp \left(-2 \pi i r t_{j}\right)\right\} \int_{0}^{1} f(t) \exp (2 \pi i r t) d t
$$

where

$$
\sum_{j=1}^{m} a_{j}=1 .
$$

This almost trivial result gives the error associated with any weighted sum of function evaluations or integration rule used to approximate an integral. It is of fundamental importance in the construction of error expressions, both for numerical quadrature and in modified form for numerical interpolation and differentiation. We refer to Eq. (2.3) as the fundamental summation formula. The usual form of Poisson's summation formula for finite ranges is a special case.

We note in passing that a much simpler proof of Eq. (2.1) than that usually given can be obtained via generalised function theory, which avoids many of the difficulties associated with the classical theory of Fourier series. Thus the Fourier expansion of the periodic delta function has the form (Lighthill [1])

$$
\sum_{m=-\infty}^{\infty} \delta\left(t-t_{j}-m\right)=1+2 \sum_{r=1}^{\infty} \cos 2 \pi r\left(t-t_{j}\right) .
$$

Poisson's summation formula follows if we multiply both sides of this equation by $f(t)$ and integrate from $-\infty$ to $\infty$. Eq. (2.1) follows if instead we replace $f(t)$ by $f(t) H(1-t) H(t)$, where $H(t)$ is the unit step function (defined in (6.4) below).

3. Particular Integration Rules. Notation. We shall find it convenient to rewrite the identity (2.3) in the abbreviated form

$$
R f-I f=E f
$$

where the integration rule $R f$ is defined by

$$
R f(x)=\sum_{j=1}^{n} a_{j} f\left(t_{j}\right) ; \quad \sum_{j=1}^{m} a_{j}=1
$$

and the exact integral If is

$$
I f=\int_{0}^{1} f(t) d t
$$

The error functional $E f$ is defined by

$$
E f=R f-I f=\sum_{r=-\infty}^{\infty} d_{r}(R) \int_{0}^{1} f(t) \exp (2 \pi i r t) d t
$$


with

$$
d_{r}(R)=\sum_{j=1}^{m} a_{j} \exp \left(-2 \pi i r t_{j}\right)=R(\exp (-2 \pi i r x))
$$

a coefficient which depends only on the rule.

In Section 4 we derive explicit expressions for $E f$ corresponding to a general rule $R f$. In this section we discuss in greater detail the form of Eq. (3.4) for certain rules.

The simplest of the conventional integration rules are those of trapezoidal type, which assign equal weight $1 / m$ to function evaluations at $m$ equally spaced points, the distance between adjacent points being $1 / \mathrm{m}$. If the first point for function evaluation is $t_{1}=1 / 2 m$, the rule is the midpoint trapezoidal rule which we write as $R^{[m, 0]}$. If the first point is $t_{1}=(1+\alpha) / 2 m,|a|<1$ we term this rule a general trapezoidal rule $R^{[m, \alpha]}$. The conventional endpoint trapezoidal rule $R^{[m, 1]}$ is obtained if we set $\alpha=1$, and remember the convention by which $f(1)$ is replaced by $\frac{1}{2}[f(0)+f(1)]$. Explicitly, we writc

$$
\begin{aligned}
& R^{[m, \alpha]} f=\frac{1}{m} \sum_{j=1}^{m} f\left(\frac{2 j-1+\alpha}{2 m}\right), \quad|\alpha|<1, \\
& R^{[m, 0]} f=\frac{1}{m} \sum_{j=1}^{m} f\left(\frac{2 j-1}{2 m}\right), \\
& R^{[m, 1]} f=\frac{1}{m} \sum_{j=1}^{m-1} f(j / m)+\frac{1}{2 m}[f(0)+f(1)] .
\end{aligned}
$$

We define $t_{\alpha}$ by

$$
R^{[1, \alpha]} f=f\left(t_{\alpha}\right), \quad t_{\alpha}=\frac{\alpha+1}{2}, \quad|\alpha|<1 .
$$

The most general rule which we consider assigns equal weight (possibly zero) to the points $x=0$ and $x=1$, and arbitrary weights to points within the integration interval. This rule may be written

$$
\mathrm{Rf}=\frac{1}{2} a_{0}[f(0)+f(1)]+\sum_{j=1}^{m} a_{j} f\left(t_{\alpha_{j}}\right)=\sum_{j=0}^{m} a_{j} R^{\left[1, \alpha_{j}\right]} f .
$$

The fundamental identity for trapezoidal rules is of the particularly simple form of the Poisson summation formula. Writing the error functional corresponding to the rule $R^{[m, \alpha]}$ by $E^{[m, \alpha]}$, we have

$$
R^{[m, \alpha]} f-\text { If }=E^{[m, \alpha]} f=\sum_{r=-\infty}^{\infty} d_{r}\left(R^{[m, \alpha]}\right) \int_{0}^{1} f(t) \exp (2 \pi i r t) d t
$$

where from Eq. (3.5)

$$
\begin{aligned}
d_{r}\left(R^{[m, \alpha]}\right) & =\frac{1}{m} \sum_{j=1}^{m} \exp (-2 \pi i r(\alpha+2 j-1) / 2 m) \\
& =\exp (-\pi i(\alpha-1) r / m), \quad \frac{|r|}{m}=\text { integer } \\
& =0 \quad \text { otherwise, }
\end{aligned}
$$


so that

$$
R^{[m, \alpha]} f-I f=\sum_{r=-\infty}^{\infty}(-1)^{r} \exp (-\pi i \alpha r) \int_{0}^{1} f(t) \exp (2 \pi i r m t) d t
$$

For the midpoint and endpoint trapezoidal rules, Eq. (3.13) take the form

$$
\begin{aligned}
& R^{[m, 0]} f-I f=\sum_{r=-\infty}^{\infty}(-1)^{r} \int_{0}^{1} f(t) \exp (2 \pi i r m t) d t \\
& R^{[m, 1]} f-I f=\sum_{r=-\infty}^{\prime} \int_{0}^{1} f(t) \exp (2 \pi i r m t) d t
\end{aligned}
$$

Other conventional rules are usually constructed to be of a specified degree. For example the Gauss-Legendre rule of degree $(2 m+1)$ which uses $m$ points for function evaluation has the form

$$
R^{[G, m]} f=\sum_{j=1}^{m} a_{j}^{[G, m]} f\left(t_{j}{ }^{[G, m]}\right)
$$

where the numbers $t_{j}{ }^{[G, m]}$ are the roots of the Legendre polynomial of degree $m$, normalised in the interval $(0,1)$, and $a_{j}{ }^{\left[{ }_{i}, m\right]}$ are related numbers. Using Eq. (3.4) we write

$$
R^{[G, m]} j-I f=\sum_{r=-\infty}^{\infty} d_{r}\left(R^{[G, m]}\right) \int_{0}^{1} f(t) \exp (2 \pi i r t) d t
$$

where

$$
d_{r}\left(R^{[G, m]}\right)=\sum_{j=1}^{m} a_{j}^{[G, m]} \exp \left(-2 \pi i r t_{j}{ }^{[G, m]}\right) .
$$

We note at this stage that the smallest argument of the Fourier transforms which occur in the sums on the right-hand sides of Eqs. (3.13) and (3.17) are $m$ and 1 respectively. In the sequel we consider methods of estimating or eliminating $E f$. From this point of view the magnitude of $E f$ is of secondary importance to its amenability to this analysis. Since an asymptotic estimate of a Fourier transform may be made more simply for large values of the argument than for small, this leads to a preference for trapezoidal rules over the Gauss-Legendre rules. The complication inherent in calculating coefficients such as those in (3.17) enhances this preference.

4. Expansions of the Euler-Maclaurin Type. The most familiar asymptotic expansion associated with the error functional of a quadrature rule is the EulerMaclaurin summation formula. This formula in its traditional form (4.23) below expresses the error functional corresponding to the endpoint trapezoidal rule in terms of the derivatives at the endpoints of the interval. It follows from the modified Poisson summation formula (3.15) in a straightforward manner. If $f(t)$ and first $w$ derivatives are continuous in $0 \leqq t \leqq 1$ and $r$ is any integer, we may integrate by parts as follows:

$$
\begin{aligned}
& \int_{0}^{1} f(t) \exp (2 \pi i r t) d t=\frac{f(1)-f(0)}{2 \pi i r}-\frac{f^{\prime}(1)-f^{\prime}(0)}{(2 \pi i r)^{2}}+\cdots \\
&+(-1)^{w} \frac{f^{(w)}(1)-f^{(w)}(0)}{(2 \pi i r)^{w+1}}+(-1)^{w+1} \int_{0}^{1} \frac{f^{(w+1)}(t) e^{2 \pi i r t}}{(2 \pi i r)^{w+1}} d t .
\end{aligned}
$$


Inserting this into the right-hand side of (3.15) and carrying out the sum over $r$ we find the traditional form (4.23) below of the Euler-Maclaurin summation formula. This derivation is due to Poisson.

We now derive a slightly more general form of this formula. Before doing so, we collect together here for convenient reference, the definitions of the Riemann zeta function, various associated functions and related results (see e.g., Whittaker and Watson [2] or Erdélyi et al. [3]). These are required both here and in our later analysis.

For $\operatorname{Re} s>1$, the Riemann zeta function $\zeta(s)$ may be defined by

$$
\zeta(s)=\sum_{r=1}^{\infty} \frac{1}{r^{s}} ; \quad \operatorname{Re} s>1 .
$$

For Re $s<1, \zeta(s)$ may be defined in terms of (4.2) using the Riemann relation

$$
\zeta(1-s)=\frac{2(s-1) !}{(2 \pi)^{s}} \cos [\pi s / 2] \zeta(s), \quad \text { all } s \neq 1 .
$$

Associated functions include

$$
t(s)=\left(1-2^{1-s}\right) \zeta(s)=\sum_{r=1}^{\infty} \frac{(-1)^{r-1}}{r^{s}}, \quad \text { Re } s>0,
$$

and the generalised zeta function

$$
\begin{aligned}
\zeta(s, a) & =\sum_{r=0}^{\infty} \frac{1}{(a+r)^{s}}, \\
\zeta(-s, a) & =\frac{2 s !}{(2 \pi)^{s+1}} \sum_{r=1}^{\infty} \frac{\sin [2 \pi a r-\pi s / 2]}{r^{1+s}}, \quad \operatorname{Re} s>0 .
\end{aligned}
$$

It is convenient to introduce a periodic generalised zeta function $\bar{\zeta}(s, a)$ defined by

$$
\bar{\zeta}(s, a)=\zeta(s, \bar{a}) ; \quad a-\bar{a}=\text { integer } \quad 0<\bar{a} \leqq 1 .
$$

The following relations are easily deduced from the above rclations

$$
\begin{aligned}
& \zeta(s, 1)=\zeta(s), \\
& \zeta\left(s, \frac{1}{2}\right)=\left(2^{s}-1\right) \zeta(s)=-\frac{2(-s) !}{(2 \pi)^{1-s}} \sin [\pi s / 2] t(1-s) .
\end{aligned}
$$

In this section and in Section $\check{\jmath}$ we need only integer values of $s$; in this case these functions and relations may be simply expressed in terms of Bernouilli polynomials and numbers. If $p$ is a positive integer

$$
\begin{aligned}
\frac{2 \zeta(2 p)}{(2 \pi)^{2 p}} & =\frac{\left|B_{2 p}\right|}{(2 p) !}, \\
\zeta(1-2 p) & =-{ }^{p} B_{2 p} / 2 p, \\
\zeta(-2 p) & =0, \quad \zeta(0)=-\frac{1}{2}, \\
\zeta(-p, a) & =-B_{1+p}(a) /(1+p) .
\end{aligned}
$$

To obtain a general form of the Euler-Maclaurin summation formula we sub- 
stitute (4.1) into (3.4). This gives for the general rule $R f$,

$$
E f=R f-\text { If }=\sum_{s=0}^{w} \frac{c_{s}(R)}{s !}\left[f^{(s)}(1)-f^{(s)}(0)\right]+\int_{0}^{1} f^{(w+1)}(t) \phi(R, t) d t
$$

where

and

$$
\frac{c_{s}(R)}{s !}=2(-1)^{s} \sum_{r=1}^{\infty} \frac{\operatorname{Re}\left[d_{r}(R) \exp (-\pi i(s+1) / 2)\right]}{(2 \pi r)^{s+1}}
$$

$$
\phi_{w+1}(R, t)=2(-1)^{w+1} \sum_{r=1}^{\infty} \frac{\operatorname{Re}\left[d_{r}(R) \exp (2 \pi i r t-\pi i(w+1) / 2)\right]}{(2 \pi r)^{w+1}} .
$$

We now evaluate the coefficients $c_{s}(R)$. We consider first the coefficient $c_{s}\left(R^{[m, \alpha]}\right) \equiv c_{s}^{[m, \alpha]}$. Substituting the expression (3.12) for $d_{r}\left(R^{[m, \alpha]}\right)$ into (4.15) and using (4.6) above, we find

$$
c_{s}^{[m, \alpha]}=-\zeta\left(-s, t_{\alpha}\right) / m^{S+1} .
$$

Thus the coefficient corresponding to the one point rule is

$$
c_{s}{ }^{[1, \alpha]}=-\zeta\left(-s, t_{\alpha}\right)
$$

Since

$$
R f=\sum a_{j} R^{\left[1, \alpha_{j}\right]} f
$$

it follows that

$$
c_{s}(R)=\sum a_{j} c_{s}^{\left[1, \alpha_{j}\right]}=-\sum a_{j} \zeta\left(-s, t_{\alpha_{j}}\right)
$$

Expansion (4.14) with these values for the coefficients constitutes a general form of the Euler-Maclaurin summation formula.

For trapezoidal type rules $R^{[m, \alpha]} f$, expression (4.20) may be replaced by its simpler form (4.17). For the endpoint and midpoint trapezoidal rules $\alpha=1$ and 0 and $t_{\alpha}=1$ and $\frac{1}{2}$ respectively. In these cases the generalised zeta function in (4.17) may be expressed in terms of zeta functions with positive integer argument using relations (4.8), (4.9), and (4.3). More precisely,

$$
\begin{gathered}
\frac{c_{s}{ }^{[m, 1]}}{s !}=-\frac{\zeta(-s, 1)}{m^{s+1} s !}=\frac{2 \zeta(s+1)}{(2 \pi m)^{s+1}} \sin [\pi s / 2] \\
\frac{c_{s}{ }^{[m, 0]}}{s !}=-\frac{\zeta\left(-s, \frac{1}{2}\right)}{m^{s+1} s !}=-\frac{2 t(s+1)}{(2 \pi m)^{s+1}} \sin [\pi s / 2] .
\end{gathered}
$$

lnserting these values into (4.14) leads to the Euler-Maclaurin summation formula in its traditional form, namely

$$
\begin{aligned}
& R^{[m, 1]} f-I f=2 \sum_{n=1}^{\infty}(-1)^{n-1} \frac{\zeta(2 n)}{(2 \pi m)^{2 n}}\left[f^{(2 n-1)}(1)-f^{(2 n-1)}(0)\right] \\
& R^{[m, 0]} f-I f=2 \sum_{n=1}^{\infty}(-1)^{n} \frac{t(2 n)}{(2 \pi m)^{2 n}}\left[f^{(2 n-1)}(1)-f^{(2 n-1)}(0)\right] .
\end{aligned}
$$

As mentioned above these relations may be derived directly from (4.1) and (3.14) without invoking the more general form (4.14). 
5. Applications to Problems of Numerical Analysis. In this section we discuss several integration rules and interpolation in order to demonstrate explicitly their fundamental connection with the identity Eq. (2.3) and the Euler-Maclaurin expansion.

(a) Integration Rules of Specified Degree. An integration rule of degree $d$ is one which integrates exactly all polynomials of degree $d$ or less. A simple example is Simpson's rule

$$
R=\frac{2}{3} R^{[m, 0]}+\frac{1}{3} R^{[m, 1]} .
$$

The degree of this rule may be derived from the expression obtained by taking the appropriate linear combination of (4.23) and (4.24). Since $2 t(2)+\zeta(2)=0$, the first term in the resulting expansion for $E f$ is zero, and the first nonzero term is that containing a factor $f^{(3)}(1)-f^{(3)}(0)$. Thus if $f(x)$ is a polynomial of degree 3 or less $E f=0$ and $R f=I f$. This establishes that the degree of Simpson's rule is at least 3 .

In general the rule

$$
R f=\sum_{j} a_{j} f\left(t_{\alpha_{j}}\right)
$$

is of degree $d$ if $a_{j}$ and $t_{\alpha_{j}}$ are chosen so that

$$
\begin{aligned}
& \sum_{j} a_{j}=1, \\
& E x^{w}=R x^{w}-I x^{w}=0 ; \quad w=1,2, \cdots, d .
\end{aligned}
$$

These equations may be written as

$$
R x^{w}=\sum_{j} a_{j} t_{\alpha_{j}}^{w}=I x^{w}=\frac{1}{w+1} ; \quad w=0,1, \cdots d .
$$

Comparison with Eq. (4.14) shows that the rule $R$ integrates all polynomials of degree $d$ or less, if and only if

$$
\sum_{j} a_{j}=1, \quad c_{s}(R)=0, \quad s=0,1, \cdots,(d-1) .
$$

The equivalence of the requirements (5.3), (5.4) may be demonstrated explicitly by means of Eqs. (4.10)-(4.18), through which Eqs. (5.4) may be replaced by

$$
\sum_{j} a_{j} B_{0}\left(t_{\alpha_{j}}\right)=1, \quad \sum_{j} a_{j} B_{1+s}\left(t_{\alpha_{j}}\right)=0, \quad s=0,1, \cdots, d-1 .
$$

Using the addition formula for Bernoulli polynomials, namely

$$
t^{w}=\frac{1}{(w+1)} \sum_{n=0}^{w}\left(\begin{array}{c}
w+1 \\
n
\end{array}\right) B_{n}(t), \quad w=0,1, \cdots,
$$

we find

$$
\begin{aligned}
R x^{w} & =\sum a_{j} t_{\alpha_{j}}^{w}=\sum_{j} a_{j} \frac{1}{(w+1)} \sum_{n=0}^{w}\left(\begin{array}{c}
w+1 \\
n
\end{array}\right) B_{n}\left(t_{j}\right) \\
& =\frac{1}{w+1} \sum_{n=0}^{w}\left(\begin{array}{c}
w+1 \\
n
\end{array}\right) \sum_{j} a_{j} B_{n}\left(t_{\alpha_{j}}\right)=\frac{1}{(w+1)}, \quad w=0,1, \cdots, d,
\end{aligned}
$$

which establishes Eq. (5.3) as a consequence of (5.4). 
The Romberg method of integration implicitly establishes a rule of specified degree in an indirect manner. This rule may be written

$$
R=\gamma_{1} R^{\left[m_{1}, 1\right]}+\gamma_{2} R^{\left[m_{2}, 1\right]}+\cdots+\gamma_{t} R^{\left[m_{\ell}, 1\right]}
$$

where $m_{1} \cdots m_{t}$ are different integers, usually $m_{i}=2^{i-1}$, and $\gamma_{i}$ are chosen so that

$$
\sum_{i=1}^{t} \gamma_{i}=1, \quad \sum_{i=1}^{t} \frac{\gamma_{i}}{m_{i}{ }^{2(8+1)}}=0, \quad s=0,1, \cdots(t-1) .
$$

Thus

$$
c_{s}(R)=\sum \gamma_{i} c_{s}^{\left[m_{i}, 1\right]}=\sum \frac{\gamma_{i}}{m_{i}^{s+1}} c_{s}^{[1,1]}=0, \quad s=0, \cdots,(2 t-1) .
$$

This follows for even values of $s$ because, by Eq. (4.21) $c_{s}{ }^{[1,1]}=0$ if $s$ is even, and for odd values of $s$ because the $\gamma_{i}$ are defined according to Eq. (5.9).

It is therefore apparent that both Gaussian and Romberg integration may be based on elimination of the early terms in the Euler-Maclaurin expansion for the error functional. It follows that in general these methods give reliable results in cases where the Euler-Maclaurin expansion is a useful expansion, but that otherwise these rules should not be expected to work well.

(b) Monte Carlo Method. In this method, $m$ values of $t_{j}$ are chosen at random and, in its most commonly used form, assigned equal weight $a_{j}=1 / m$. Estimation of the error term then becomes a problem in the theory of stochastic processes. The statistical properties of the error functional are intimately connected with Pearson's random walk problem. The coefficients $d_{r}(R)$ which occur in the identity Eq. (3.4) for this rule are

$$
d_{r}(R)=\sum_{j=1}^{m} a_{j} \exp \left(-2 \pi i r t_{j}\right)=\rho \exp (2 \pi i \Phi(r)) .
$$

The modulus of this sum is precisely the distance travelled after $m$ steps by a twodimensional random walker, who takes steps of length $a_{1}, a_{2}, \cdots, a_{m}$ at angles $\theta_{1}=2 \pi t_{1}, \theta_{2}=2 \pi t_{2}, \cdots, \theta_{m}=2 \pi t_{m}$ between successive directions and the $x$ axis. The distribution of distances travelled in $m$ steps is that of $\rho\left(\theta_{1}, \cdots, \theta_{m}\right)$ which results from allowing each $\theta_{j}$ to take on any value between 0 and $2 \pi$ with equal probability. The solution to this problem is well known (see, e.g. Watson [4]), and the probability distribution $P(s)=\operatorname{Pr}\{\rho<s\}$ is given by Kluyuer's formula which yields

$$
P(s)=\operatorname{Pr}\{\rho<s\}=s \int_{0}^{\infty} J_{1}(s \nu) \prod_{j=1}^{m} J_{0}\left(\nu a_{j}\right) d \nu
$$

where $J_{0}$ and $J_{1}$ are Bessel functions.

The statistical properties of $\rho$ are independent of $r$. In the special case when all the step lengths are equal, $a_{j}=1 / m$; if $m$ is large, the important part of the integrand of Eq. (5.12) is that for which $\nu$ is small. For such values of $\nu$ we have

$$
J_{0}(t) \approx \exp \left(-t^{2} / 4\right)
$$


Using Weber's first exponential integral, Watson [4, p. 393], we find that the density function is

$$
\begin{aligned}
\frac{d P(s)}{d s} & =\int_{0}^{\infty} \nu s J_{0}(\nu s)\left(J_{0}\left(\frac{\nu}{m}\right)\right)^{m} d \nu \\
& \approx \int_{0}^{\infty} \nu s J_{0}(\nu s) \exp \left(-\nu^{2} / 4 m\right) d \nu=2 m s e^{-m s^{2}}
\end{aligned}
$$

which is a Gaussian whose expected value is $(\sqrt{ } \pi / 2) m^{-1 / 2}$.

The expression for the error term then becomes

$$
R f-I f=\sum_{r=-\infty}^{\infty}\left|d_{r}(R)\right| \exp (2 \pi i \Phi(r)) \int_{0}^{1} f(t) e^{2 \pi i r t} d t
$$

where $\rho=\left|d_{r}(R)\right|$ is a random variable whose distribution is given by Eq. (5.14).

(c) Interpolation and Differentiation. These topies may also be treated in a similar manner. Choosing weights $a_{j}$ so that

$$
a_{0}=-\sum_{j=1}^{m} a_{j}=1
$$

it follows that

$$
f\left(t_{0}\right)-\sum_{j=1}^{m} a_{j} f\left(t_{j}\right)=2 \sum_{r=1}^{\infty} \sum_{j=0}^{m} a_{j} \int_{0}^{1} f(t) \cos 2 \pi r\left(t-t_{j}\right) d t .
$$

This gives the error associated with the most general interpolation formula. If we integrate by parts successively, and perform the sums over $r$ using Eqs. (4.6) and (4.13), we have

$$
\begin{aligned}
f\left(t_{0}\right)- & \sum_{j=1}^{m} a_{j} f\left(t_{j}\right) \\
& =-\sum_{j=0}^{m} a_{j}\left\{[f(1)-f(0)] B_{1}\left(t_{j}\right)+\left[f^{\prime}(1)-f^{\prime}(0)\right] B_{2}\left(t_{j}\right)+\cdots\right\} .
\end{aligned}
$$

If the interpolation formula

$$
f\left(t_{0}\right) \simeq \sum_{j=1}^{m} a_{j} f\left(t_{j}\right)
$$

is to be exact whenever $f(x)$ is any polynomial of degree $d$ or less, the coefficients of $\left[f^{(s)}(1)-f^{(s)}(0)\right] ; s<d$, in (5.18) must be zero. This implies that $a_{j}$ and $t_{j}$ satisfy the set of equations

$$
\sum_{j=1}^{m} a_{j} B_{n}\left(t_{j}\right)=B_{n}\left(t_{j}\right), \quad n=0,1, \cdots(d-1),
$$

whose solution gives the Lagrange coefficients. A number of other interpolation formulae may be considered using similar techniques. A similar method can be used to handle differentiation.

6. Lighthill's Procedure. If $f(x)$ or its early derivatives are discontinuous in the interval $0 \leqq x \leqq 1$, the theory of Sections $3,4,5(\mathrm{a})$ and $5(\mathrm{c})$ is no longer valid. 
The conventional Euler-Maclaurin series is not an infinite asymptotic expansion and has to be terminated by an error remainder term. However, the theory of Section 2 is independent of whether or not $f(x)$ or its derivatives are continuous. We therefore return to Section 2 and develop asymptotic expansions more suited to computational purposes, in those cases for which $f(x)$ has simple algebraic or algebraico-logarithmic singularities on the integration interval. We now consider an integrand $f(x)$ of the form

$$
f(x)=x^{\beta}(1-x)^{\omega}\left|x-t_{k}\right|^{\gamma} \operatorname{sgn}\left(x-t_{l}\right)\left|x-t_{l}\right|^{\delta} h(x)
$$

where $h(x)$ and its derivatives are continuous in the interval $0 \leqq x \leqq 1$, and $\beta$, $\gamma, \delta$, and $\omega$ are not integers.

To proceed further we require an asymptotic expansion for the Fourier transform

$$
g(r)=\int_{0}^{1} f(t) \exp (-2 \pi i r t) d t
$$

with $f(x)$ given by Eq. (6.1). The expansion Eq. (4.1) which was obtained by integration by parts is not valid for this function.

Although appropriate asymptotic expansions with a remainder term exhibited explicitly can be found by transform techniques, we find it convenient to appeal to the powerful, simple and systematic method of Lighthill [1] who uses generalised function theory. The result is the expansion (6.15) below. We restate here the prescription developed by Lighthill, together with the theorem which justifies this prescription.

The Fourier transform required is that of the generalised function

$$
\phi(x)=f(x) H(x) H(1-x)
$$

where $H(x)$ is the step function defined by

$$
\begin{array}{rr}
H(x)=0, & x<0, \\
\frac{1}{2}, & x=0, \\
1, & x>0 .
\end{array}
$$

$\phi(x)$ coincides with $f(x)$ in the interval of integration, and is zero elsewhere. The behaviour of the Fourier transform

$$
g(r)=\int_{-\infty}^{\infty} \phi(t) \exp (-2 \pi i r t) d t
$$

depends critically on the behaviour of $\phi(x)$ in the neighbourhoods of its singularities. Let these lie at $x=t_{0}, t_{1}, t_{2}, \cdots, t_{m}$ where $t_{0}=0$ and $t_{1}=1$. Suppose further that corresponding to each singularity $t_{j}$ we construct an 'approximating' function $F_{j}(x)$ with the following properties:

(6.6) (i) $\phi(x)-F_{j}(x)$ has an absolutely integrable $N$ th derivative in a neighbourhood of $x=t_{j}$.

(6.7) (ii) $F_{j}(x)$ is a linear combination of functions of the types $\left|x-t_{j}\right|^{\alpha}$, $\left|x-t_{j}\right|^{\alpha} \operatorname{sgn}\left(x-t_{j}\right),\left|x-t_{j}\right|^{\alpha} \ln \left|x-t_{j}\right|$ and $\left|x-t_{j}\right|^{\alpha} \ln \left|x-t_{j}\right| \operatorname{sgn}\left(x-t_{j}\right)$ for different values of $\alpha$.

THEOREn. If $F_{j}(x), j=0 \cdots m$ satisfy these conditions, and have as their Fourier 
transforms $G_{j}(r)$;

$$
G_{j}(r)=\int_{-\infty}^{\infty} F_{j}(t) \exp (-2 \pi i v t) d t
$$

Then an asymptotic expansion for $g(r)$ is

$$
g(r)=\sum_{j=0}^{m} G_{j}(r)+O\left(|r|^{-N}\right) \text { as } \quad|r| \rightarrow \infty .
$$

This theorem provides a method for writing down an asymptotic expansion for the Fourier transform of any function with singularities of the type (6.7) above. We need to know only the Fourier transforms of functions of that type, which are listed by Lighthill [1, p. 43].

The singularities of $f(x)$ defined by Eq. (6.1) lie at $0,1, t_{k}$ and $t_{l}$. We obtain the required approximating functions as follows. We define functions $\psi_{j}(x)$, which are continuous and have continuous derivatives at $x=t_{j}$, by

$$
\begin{aligned}
f(x) & =x^{\beta} \psi_{0}(x)=(1-x)^{\omega} \psi_{1}(x)=\left|x-t_{k}\right|^{\gamma} \psi_{k}(x) \\
& =\left|x-t_{l}\right|^{\delta} \operatorname{sgn}\left(x-t_{l}\right) \psi_{l}(x) .
\end{aligned}
$$

The approximating functions $F_{j}(x)$ are constructed from $\psi_{j}(x)$ by retaining only the first $N$ terms in the power series expansion of $\psi_{j}(x)$ about $x=t_{j}$. Thus

$$
\begin{aligned}
& F_{0}(x)=\sum_{s=0}^{N-1} \frac{\psi_{0}^{(s)}(0)}{s !} x^{\beta+s} H(x) \\
& F_{1}(x)=\sum_{s=0}^{N-1}(-1)^{s} \frac{\psi_{1}^{(s)}(1)}{s !}(1-x)^{\omega+s} H(1-x) \\
& F_{k}(x)=\sum_{s=0}^{N-1} \frac{\psi_{k}^{(s)}\left(t_{k}\right)}{s !}\left|x-t_{k}\right|^{\gamma+s}\left[\operatorname{sgn}\left(x-t_{k}\right)\right]^{s} \\
& F_{1}(x)=\sum_{s=0}^{N-1} \frac{\psi_{l}^{(s)}\left(t_{1}\right)}{s !}\left|x-t_{l}\right|^{\delta+s}\left[\operatorname{sgn}\left(x-t_{l}\right)\right]^{s+1}
\end{aligned}
$$

These functions satisfy conditions (i) and (ii). The Fourier transforms of individual terms are given by Lighthill $[1$, p. 43]. The results we require are listed below. In terms of a function

$$
h(\beta, r)=\frac{\beta !}{(2 \pi i r)^{\beta+1}}
$$

we have

$$
\begin{gathered}
\int_{-\infty}^{\infty} x^{\beta+s} H(x) \exp (-2 \pi i r x) d x=h(\beta+s, r), \\
\int_{-\infty}^{\infty}(1-x)^{\omega+s} H(1-x) \exp (-2 \pi i r x) d x=h(\omega+s,-r), \\
\int_{-\infty}^{\infty}\left|x-t_{k}\right|^{\gamma+s} e^{-2 \pi i r x} d x=\exp \left(-2 \pi i t_{k} r\right)[h(\gamma+s, r)+h(\gamma+s,-r)], \\
\int_{-\infty}^{\infty}\left|x-t_{l}\right|^{\delta+s} \operatorname{sgn}\left(x-t_{l}\right) e^{-2 \pi i r x} d x \\
=\exp \left(-2 \pi i t_{l} r\right)[h(\delta+s, r)-h(\delta+s,-r)] .
\end{gathered}
$$


Substitution of these results into Eq. (6.10) yields

$$
\begin{aligned}
& \int_{0}^{1} f(x) \exp (-2 \pi i r x) d x=\sum_{s=0}^{N-1} \frac{1}{s !}\left\{\psi_{0}^{(s)}(0) h(\beta+s, r)\right. \\
& +(-1)^{s} \psi_{1}^{(s)}(1) h(\omega+s,-r) \\
& +\exp \left(-2 \pi i t_{k} r\right) \psi_{k}^{(s)}\left(t_{k}\right)[h(\gamma+s, r)+h(\gamma+s,-r)] \\
& \left.+\exp \left(-2 i t_{1} r\right) \psi_{1}^{(s)}\left(t_{1}\right)\left[h(\delta+s, r)-(-1)^{s} h(\delta+s,-r)\right]\right\}+O\left(|r|^{-N}\right) .
\end{aligned}
$$

This is the principal result of this section and provides the basis for our subsequent analysis. We note that while the generalised functions on the right-hand side coincide with, and can therefore be interpreted in terms of, ordinary functions, the derivation of Eq. (6.15) rests on generalised function theory. For example, the final equation of the set (6.14) is meaningless if interpreted in terms of ordinary functions for most values of $\delta$.

7. The Euler-Maclaurin Formula for Endpoint Singularities. We now use this result to find an asymptotic expansion for Ef associated with

$$
f(x)=x^{\beta}(1-x)^{\omega} h(x),
$$

where $h(x)$ and its derivatives are continuous within the interval of integration, and consider in detail an arbitrary trapezoidal rule $R^{[m, \alpha]},|\alpha|<1$ Eq. (3.13) asserts that

$$
E^{[m, \alpha]} f=R^{[m, \alpha]} f-I f=\sum_{r=-\infty}^{\infty}(-1)^{r} \exp (-\pi i r \alpha) \int_{0}^{1} f(t) \exp (2 \pi i r m t) d t .
$$

The integral on the right-hand side has the asymptotic expansion given by (6.15). Since $f(x)$ has endpoint singularities only the first two terms contribute, and substitution into (7.2) yields

$$
E^{[m, \alpha]} f=\sum_{s=0}^{N-1} \frac{\psi_{0}^{(s)}(0)}{s !} c_{s}^{[m, \alpha]}(0, \beta)+\sum_{s=0}^{N-1} \frac{\psi_{1}^{(s)}(1)}{s !} d_{s}^{[m, \alpha]}(1, \omega)+O\left(m^{-N}\right) .
$$

The coefficients in this expansion have the values

$$
\begin{aligned}
& c_{s}{ }^{[m, \alpha]}(0, \beta)=\sum_{r=-\infty}^{\infty}(-1)^{r} \exp (-\pi i r \alpha) \frac{(\beta+s) !}{(-2 \pi i r m)^{\beta+s+1}} \\
& =\frac{\bar{\zeta}\left(-\beta-s, t_{\alpha}\right)}{m^{\beta+s+1}} ; \quad \quad \beta>-1,|\alpha|<1,
\end{aligned}
$$

where we have used expression (6.13) for $h(\beta+s, r)$ and Eq. (4.6) for the generalised zeta function. Similarly

$$
d_{s}^{[m, \alpha]}(1, \omega)=\frac{(-1)^{s}}{m^{\omega+s+1}} \bar{\zeta}\left(-\omega-s, 1-t_{\alpha}\right), \quad \omega>-1,|\alpha|<1 .
$$

For the special case of an endpoint trapezoidal rule for which $\alpha=1$, we have

$$
\begin{array}{rlrl}
c_{s}{ }^{[m, 1]}(0, \beta) & =(-1)^{s} d_{s}{ }^{[m, 1]}(1, \beta)=\frac{\zeta(-\beta-s)}{m^{\beta+s+1}}, & s>0 ; \beta & \geqq 0, \\
& =0, & s=\beta=0 .
\end{array}
$$


Inserting these values of $c_{s}$ and $d_{s}$ into (7.3) gives the asymptotic expansion for the error functional associated with the rule $R^{[m, \alpha]}$.

The corresponding expansion for an arbitrary rule may be obtained as in Section 4 by taking appropriate linear combinations of the expansions corresponding to the one point rules $R^{[1, \alpha]}$. The coefficients in this expansion involve linear combinations of generalised zeta functions and we do not write it out here. We give explicitly the expansions corresponding to the much simpler midpoint and endpoint trapezoidal rules. These are

$$
\begin{aligned}
R^{[m, 0]} f-\text { If }= & \sum_{s=0}^{N-1} \frac{\psi_{0}^{(s)}(0)}{s !} \frac{\zeta\left(-\beta-s, \frac{1}{2}\right)}{m^{\beta+s+1}} \\
& +\sum_{s=0}^{N-1} \frac{(-1)^{s} \psi^{(s)}(1) \zeta\left(-\omega-s, \frac{1}{2}\right)}{s ! m^{\omega+s+1}}+O\left(m^{-N}\right) \\
R^{[m, 1]} f-\text { If }= & \sum_{s=s_{0}}^{N-1} \frac{\psi_{0}^{(s)}(0)}{s !} \frac{\zeta(-\beta-s)}{m^{\beta+s+1}} \\
& +\sum_{s=s_{1}}^{N-1} \frac{(-1)^{s} \psi_{1}^{(s)}(1) \zeta(-\omega-s)}{s ! m^{(\beta+s+1)}}+O\left(m^{-N}\right)
\end{aligned}
$$

where $s_{0}, s_{1}=0$ unless $\beta, \omega=0$ in which case $s_{0}, s_{1}=1$. Both expansions (7.7) and (7.8) reduce to the usual forms (4.23), (4.24) if $\beta=\omega=0$ or if $\beta$ and $\omega$ are both integers. The generalised zeta functions which occur here may be expressed in terms of zeta functions of positive argument using Eqs. (4.3) and (4.9). An integral representation for the remainder term in these expansions (7.7), (7.8) for the particular case that one of the exponents $\beta$ or $\omega$ is an integer was first given by Navot $[5]$.

Expression (7.3) for an arbitrary trapezoidal rule has the form

$$
R^{[m, \alpha]} f-I f=\sum_{s=0}^{N-1} \frac{a_{s}}{m^{\beta+s+1}}+\sum_{s=0}^{N-1} \frac{b_{s}}{m^{\omega+s+1}}+O\left(m^{-N}\right)
$$

where $a_{s}$ and $b_{s}$ are independent of $m$. Thus a modified form of Romberg integration is possible, based on the values of $R^{[m, \alpha]} f$ for different values of $m$. Eqs. (7.7) and (7.8) are special cases with $\alpha=0$ or 1 respectively. These have the marginal advantage that the coefficients involve zeta functions rather than generalised zeta functions. If the expansions are used directly it is easier to calculate ordinary zeta functions or, as a rough guide, to estimate their magnitude.

Logarithmic Singularities. If we consider a function $f(x)$ with integrable logarithmic singularities, for example

$$
f(x)=x^{\beta} \ln x(1-x)^{\omega} h(x),
$$

the appropriate generalisation of the Euler-Maclaurin formula may be derived in precisely the same way. However, it is simpler to differentiate Eqs. (7.3), (7.7) and (7.8) directly.

If we differentiate Eq. (7.9) with respect to $\beta$, and multiply by $\beta$ we have

$$
R^{[m, \alpha]} f-I f=\sum_{s=0}^{N-1} \frac{e_{s}+a_{s} \ln m}{m^{\beta+s+1}}+\sum_{s=0}^{N-1} \frac{b_{s}}{m^{\omega+s+1}}+O\left(m^{-N}\right)
$$


where $f$ now represents the function of Eq. (7.10) and not that of (7.1) and $a_{s}$, $b_{s}$ and $e_{s}$ are independent of $m$. The coefficients $b_{s}, a_{s}$ are the same as those of Eq. (7.9) but $e_{s}$ involves derivatives of zeta functions. The application of Romberg methods, based on (7.11) is in principle possible, but clearly rather more complicated than in the case involving only algebraic endpoint singularities.

Similarly the method may be extended to functions with singularities of the form $\left|x-t_{k}\right|^{\alpha}\left(\ln \left|x-t_{k}\right|\right)^{n}$ and $\left|x-t_{l}\right|^{\alpha}\left(\ln \left|x-t_{l}\right|\right)^{n} \operatorname{sgn}\left(x-t_{l}\right)$.

8. The Generalised Euler-Maclaurin Expansion. We now deal with the more general function of Section 6 ,

$$
f(x)=x^{\beta}(1-x)^{\omega}\left|x-t_{k}\right|^{\gamma}\left|x-t_{l}\right|^{\delta} \operatorname{sgn}\left(x-t_{l}\right) h(x) .
$$

Again we confine ourselves to stating the result for $R^{[m, \alpha]}$ the trapezoidal rule. As before the corresponding expansions for an arbitrary rule may be obtained by taking linear combinations of $R^{[1, \alpha]}$ with different values of $\alpha$. The same procedure used in Section 7 gives

$$
\begin{aligned}
E^{[n, \alpha]} f= & R^{[m, \alpha]} f-I f \\
= & \sum_{s=0}^{N-1} \frac{1}{s !}\left\{\psi_{0}^{(s)}(0) c_{s}^{[m, \alpha]}(0, \beta)+\psi_{1}^{(s)}(1) d_{s}^{[m, \alpha]}(1, \omega)\right. \\
& +\psi_{k}^{(s)}\left(t_{k}\right)\left[c_{s}^{[m, \alpha]}\left(t_{k}, \gamma\right)+d_{s}^{[m, \alpha]}\left(t_{k}, \gamma\right)\right] \\
& \left.+\psi_{l}^{(s)}\left(t_{l}\right)\left[c_{s}{ }^{[m, \alpha]}\left(t_{l}, \delta\right)-d_{s}^{[m, \alpha]}\left(t_{l}, \delta\right)\right]\right\} \\
& +O\left(m^{-N}\right), \quad|\alpha|<1 .
\end{aligned}
$$

where

$$
\begin{aligned}
& c_{s}^{[m, \alpha]}\left(t_{k}, \beta\right)=\bar{\zeta}\left(-\beta-s, t_{\alpha}-m t_{k}\right) / m^{\beta+s+1}, \\
& d_{s}^{[m, \alpha]}\left(t_{k}, \beta\right)=(-1)^{s} \bar{\zeta}\left(-\beta-s, m t_{k}-t_{\alpha}\right) / m^{\beta+s+1} .
\end{aligned}
$$

The expansion for functions with logarithmic singularities follows by differentiation with respect to the appropriate parameter.

It is important to note that when singularities occur within the interval, and not only at the endpoints, the constants $a_{s}$ and $b_{s}$ in the equation corresponding to (7.9) contain parameters which depend on $m$, through terms such as $\bar{\zeta}(-s-\beta$; $\left.t_{\alpha}-m t_{k}\right)$. Consequently it would be extremely complicated to use expansion (8.1) as a basis for Romberg integration.

9. Behaviour near Rule Singularities. Finally we describe briefly the principal features of the asymptotic expansion $E f=R f-I f$ in the case that the rule $R f$ requires a function evaluation $f\left(t_{j}\right)$ at a point $t_{j}$ which is close to a singularity $t_{k}$ of $f(x)$. We confine ourselves to considering a simple example, which illustrates adequately the general situation without involving any complicated algebra. Thus we set

$$
f(x)=\left|x-t_{k}\right|^{\gamma}, \quad-\frac{1}{2}<\gamma<0,
$$

and consider

$$
R^{[m, 1]} f-I f=E^{[m, 1]} f
$$


in the case that

$$
t_{j}=j / m=t_{k}-\epsilon
$$

and

$$
0<\epsilon \ll \frac{1}{m}
$$

In this case, the principal contribution to the rule sum is $(1 / m) f\left(t_{j}\right)$ and

$$
R^{[m, 1]} f=\frac{\epsilon^{\gamma}}{m}+\text { small terms. }
$$

The integral If is not large. The asymptotic expansion (8.1) for $E^{[m, 1]} f$ is simple to write down. We find

$$
\begin{aligned}
\psi_{0}(x) & =\psi_{1}(x)=f(x), \\
\psi_{k}(x) & =1, \\
c_{0}^{[m, 1]}\left(t_{k}, \gamma\right) & =\zeta(-\gamma, m \epsilon) / m^{\gamma+1}, \\
d_{0}^{[m, 1]}\left(t_{k}, \gamma\right) & =\zeta(-\gamma, 1-m \epsilon) / m^{\gamma+1} .
\end{aligned}
$$

Substituting these values into (8.1) it follows

$$
\begin{aligned}
E^{(m, 1]} f= & \sum_{s=1}^{N-1} \frac{\zeta(-s)}{s ! m^{s+1}}\left\{\psi_{0}^{(s)}(0)+(-1)^{s} \psi_{1}^{(s)}(1)\right\} \\
& +\frac{\zeta(-\gamma, m \epsilon)+\zeta(-\gamma, 1-m \epsilon)}{m^{\gamma+1}} .
\end{aligned}
$$

Except for the final term, this expansion is identical to the traditional Euler-Maclaurin summation formula (4.24) in the case that $f(x)$ has no singularity. The size of this final term may be estimated using Eq. (4.5) in the form

$$
\zeta(-\gamma, m \epsilon)=(m \epsilon)^{\gamma}+\zeta(-\gamma, 1+m \epsilon)
$$

and the circumstance that $\zeta(-\gamma, 1)$ is small when $0<-\gamma<+\frac{1}{2}$. Thus this single term in $E^{[m, 1]} f$ is responsible for a contribution $\epsilon^{\gamma} / m$ which equals the single large term in $R^{[m, 1]} f$.

The above discussion indicates something more than merely that the identity (8.1) $R f-I f=E f$ is reasonable in this case. It shows that the effect of an unreasonably large value of $R f$, resulting from a function evaluation near a singularity, is exactly balanced by the first term in that part of the expansion for $E f$ which arises from the existence of that singularity. Thus the formula $R f-I f=E f$ may be quite suitable for numerical computation of $I f$ even if $R f$ is a completely unrealistic approximation to $I f$. Its suitability depends principally on the general behaviour of the asymptotic expansion for $E f$, and the magnitude of $E f$ is not a particularly useful criterion.

10. Conclusion. After this work was completed, our attention was drawn to the important work of Navot [5], [6], [7] and of Waterman et al. [8]. The principal expansion given by us (8.1) constitutes a generalisation of previous work in that 
more than one singularity is allowed and a general quadrature rule is also allowed. However, the previous work provided a truncation error term in the simpler cases, and considered also the effect of an essential singularity. The methods of proof in this work are quite different from those in previous work, and we believe somewhat simpler.

Our intention has been to develop the theory of numerical quadrature entirely from the point of view of the asymptotic expansion of the error functional, in a manner in which most of the results are obtained in a straightforward manner. Various specific parts of the theory of numerical quadrature usually forming watertight compartments such as rules of high degree, the Monte Carlo method, methods for dealing with singularities and methods of extrapolation fall into place naturally as the theory is developed. Knowledge of the appropriate asymptotic expansion also indicates clearly why particular methods of quadrature are likely to be unsuccessful in particular cases.

Acknowledgments. One of the authors (B. W. N.) acknowledges the support of the Australian Commonwealth Government through the award of a Queen Elizabeth II Post Doctoral Fellowship. We are grateful to Professor J. M. Blatt for a critical discussion and to the referee for many helpful suggestions.

Department of Applied Mathematics

The University of New South Wales

Kensington, N.S.W., Australia

1. M. J. Lighthill, Introduction to Fourier Analysis and Generalised Functions, Cambridge Univ. Press, New York, 1958; 1960. MR 19, 1066; MR 22 \#5888.

2. E. T. Whittaker \& G. N. Watson, A Course of Modern Analysis, Cambridge Univ. Press, New York, 1958.

3. A. Erdélyi, W. Magnus, F. Oberhettinger \& F. G. Tricomi, Higher Transcendental Functions, Vol. 1, (Calif. Inst. Tech. Bateman Manuscript Project), Chapter 1, McGraw-Hill, New York, 1953, MR 15, 419. 419 .

4. G. N. WATson, Theory of Bessel Functions, Cambridge Univ. Press, New York, 1958, p.

5. I. N.Avot, "An extension of the Euler-Maclaurin summation formula to functions with a branch singularity," J. Math. and Phys., v. 40, 1961, pp. 271-276. MR $25 \# 4290$.

6. I. NAVOT, "A further extension of the Euler-Maclaurin summation formula," J. Math. and Phys., v. 41, 1962 , pp. 155-163.

7. I. Nivot, "The Euler-Maclaurin functional for functions with a quasi-step discontinuity," Math. Comp. v. 17, 1963, pp. 337-345. MR 27 \#5363.

8. P. C. WATERMAN, J. M. YOS \& R. J. ABODEELY, "Numerical integration of non-analytic functions," J. Math. and Phys., v. 43, 1964, p. 45-50. 